\title{
Teaching Organic Electronics: The Synthesis of the Conjugated Polymer MEH-PPV in a Hands-on Experiment for Undergraduate Students
}

\author{
Amitabh Banerji ${ }^{1 * *}$, Ann-Kathrin Schönbein ${ }^{2}$, Lena Halbrügge ${ }^{1}$ \\ ${ }^{1}$ Institute of Chemistry Education, University of Cologne, Cologne, Germany \\ ${ }^{2}$ Department of Molecular Electronics, Max Planck Institute for Polymer Research, Mainz, Germany \\ *Corresponding author: a.banerji@uni-koeln.de
}

\begin{abstract}
Organic light emitting diodes (OLEDs) are modern illuminants of the next generation. OLEDs use (among others) semiconducting polymers for light emission and open the pathway to innovative applications as flexible or transparent displays or luminaire. For the school-implementation of OLEDs low-cost experiments and teaching materials have been developed earlier. This contribution delivers a school-experiment for the synthesis of a semiconducting polymer and presents a successful example of a curricular innovation based on the cooperation between subject science and science education.
\end{abstract}

Keywords: OLED, conjugated polymers, organic semiconductors, MEH-PPV, future technology

Cite This Article: Amitabh Banerji, Ann-Kathrin Schönbein, and Lena Halbrügge, "Teaching Organic Electronics: The Synthesis of the Conjugated Polymer MEH-PPV in a Hands-on Experiment for Undergraduate Students.” World Journal of Chemical Education, vol. 6, no. 1 (2018): 54-62. doi: 10.12691/wjce-6-1-9.

\section{Introduction}

Electronics, whose functional elements are based on semiconducting organic materials, are related to organic electronics. This is no longer a vision; it's an inherent part of our everyday life. This kind of electronics is already being used in innovative products such as transparent OLED-displays (organic light emitting diode) or flexible and lightweight OPV-cells (organic photovoltaic). OLEDs and OPVs use special polymers, oligomers or small molecules instead of inorganic semiconductors like silicon or gallium-arsenide etc. The common structural characteristic of all organic semiconductors is a continuous conjugated $\pi$-electron system. To teach Organic Electronics on an undergraduate level, we have developed a teaching concept along with a material kit [1,2] containing materials to build and examine OLED- and OPV-devices in a low-cost approach without cleanroom facilities. This material kit contains the commercially available organic semiconductors Superyellow (for OLED) and P3HT/PCBM (for OPV). In cooperation with the Max-Planck-Institute for Polymer Research in Mainz (Department of Molecular Electronics), we have now succeeded in developing a "quick and easy" experiment for the synthesis of the semiconducting polymer MEH-PPV (Poly[2-methoxy-5-(2'-ethylhexyloxy)1,4-phenylene vinylene]). Furthermore, we could incorporate the self-made polymer into the low-cost OLED resulting in a bright orange-red electroluminescent device. This extension of the OLED-teaching-experiment will help learners to understand the structure-property relationships of the applied polymers better and emphasize the role of chemistry in the field of organic electronics. Additionally, the synthesis of MEH-PPV correlates to mandatory contents of the chemistry syllabus (e.g. dyes, polymer synthesis, solubility, intermolecular interactions, etc.) and enables educators to teach these contents with an innovative context [3].

Parts of the following contribution have been published by us in CHEMKON [4] (a German journal for Chemistry Education). The aim of this article is to make our results internationally accessible and to present the recent developments in the quick and easy synthesis of MEH-PPV.

\section{Scientific Background}

Since Heeger, MacDiarmid and Shirakawa received the Nobel Prize in Chemistry in 2000 for their work on semiconducting polymers, the interest in this class of materials has increased steadily. In 1977, they changed the classic understanding of polymers as insulators and created „The fourth generation of polymeric materials“ [5]. Heeger, MacDiarmid and Shirakawa doped polyacetylene (PAC) (chemically and electrochemically) and transferred the bad semiconductor PAC into the metallic state [6]. The characteristic feature of semiconducting polymers is their conjugated backbone. Each $\mathrm{p}_{\mathrm{z}}$-orbital at the carbon atoms is occupied by one electron, which leads to an overlap of $\pi$ - and $\pi^{*}$-moleculuar orbitals. The more carbon atoms are conjugated, the more splitting of these molecular orbitals takes place, until a kind of band structure, similar to metals, results (see Figure 1). Free charge carriers (electrons and so-called holes) can move along the polymer backbone in these bands. 


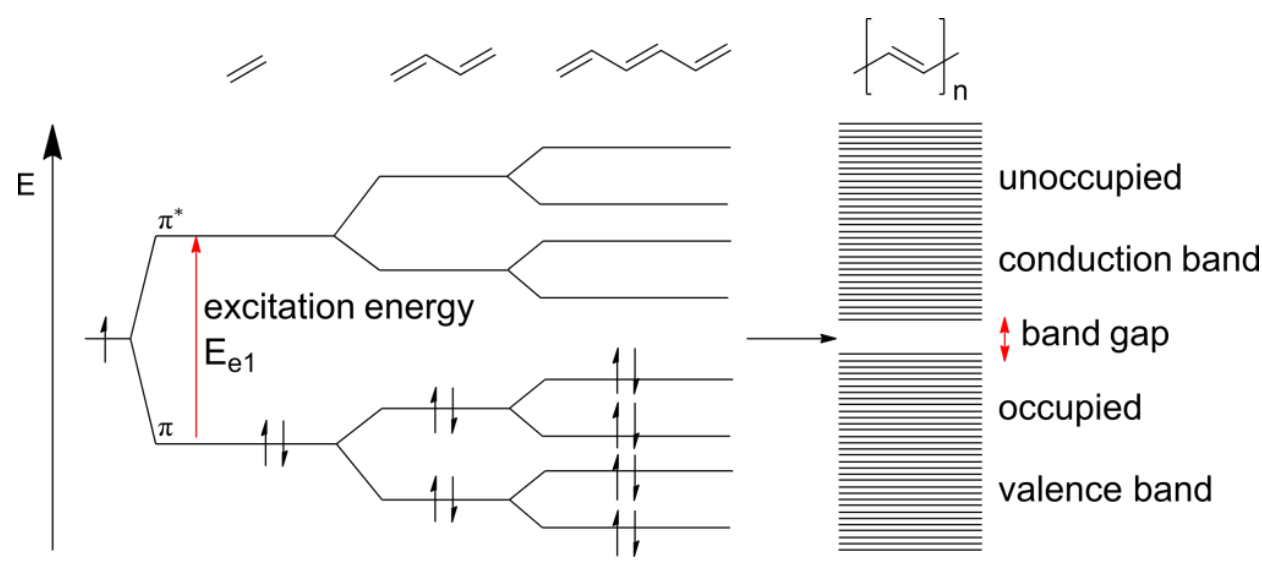

Figure 1. Increasing splitting of the $\pi$ and $\pi^{*}$-molecular orbitals with increasing conjugation. For the case of a conjugated polymer in the ground state a fully occupied valence and an empty conduction band results, in which charge carriers can move

A band gap emerges between the valence and conduction band, which is typically smaller than $3 \mathrm{eV}$ for semiconductors. In the case of organic semiconductors, the HOMO (Highest Occupied Molecular Orbital) and LUMO (Lowest Unoccupied Molecular Orbital) energies define the band gap. The energy levels mainly depend on the chemical structure (planar or partially twisted), electron withdrawing (EWG) or donating (EDG) substituents and the effective conjugation length [7]. In the case of polymers, the effective conjugation length is limited to max. $6 \mathrm{~nm}$, which typically corresponds to 10-15 monomer repeat units [8]. The bigger the energetic distance (band gap) between the HOMO and LUMO, the shorter is the effective conjugation length over which charge carriers are actually conjugated.

The field of organic electronics does not focus only on polymers. In the 1980s Tang and Slyke [9] as well as Adachi, Tsutsui and Saito [10] built the first so-called small molecule (SM) based OLEDs. Small molecules are also fully conjugated systems, which are usually, due to a lack of solubilizing side chains, very limited in solubility or not soluble at all. This makes the processing of such materials very expensive, since the compounds have to be evaporated in vacuum chambers, which additionally limits the size of the active area in e.g. state-of-the-art TV displays. Nowadays, the focus in organic electronics is mainly on materials, which can be processed from solution, which would make roll-to-roll-printing and therefore cheap large-area applications possible [11]. The important cornerstone for solution-processed OLEDs was laid in 1990 by Friend et al. [12], who observed electroluminescence in Poly( $p$-phenylene vinylene) (PPV) for the first time. As polymers are known to have excellent film forming properties, when they are processed from solution, a whole new field of research started. By attaching suitable solubilizing side-chains to the polymer(s), not only the processing could be improved, but also the band gap could be adjusted. Instead of a complex procedure including evaporation, the devices were now fabricated by spin coating, dip coating or printing from common organic solvents.

In Figure 2, two classical organic semiconductors are shown, which have already been applied in OLEDs. In the case of the SM, usually metal complexes containing heavy metal atoms, like tris[2-phenylpyridinato- $\left.C^{2}, N\right]$ iridium(III) (1), are used. Due to the fact that unsubstituted PPV is not soluble and meltable, the alkoxy-substituted poly[2methoxy-5-(2'-ethylhexyloxy)-1,4-phenylene vinylene] (MEH-PPV, 2) is shown here, which till date is a work horse polymer in organic electronics and one of the best studied organic semiconductors in the world [13]. Also in this work, MEH-PPV will be the model substance for a polymeric semiconductor.<smiles></smiles>

1

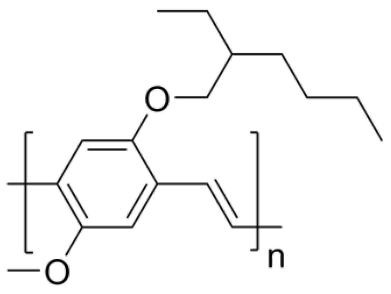

MEH-PPV
Figure 2. Selected representative organic semiconductors, which are applied in OLEDs: Small molecule tris[2-phenylpyridinato- $C^{2}$, $N]$ iridium(III) (1), (left) and work horse polymer MEH-PPV 2 (right)

\section{Synthesis of Poly( $p$-phenylene vinylene)s}

PPVs can be synthesized using different approaches. In the 1960s the first PPVs were made using the Wittig and later the Wittig Horner reaction [14]. Besides these metalfree polymerizations, the classical C-C-cross coupling reactions, like the Suzuki polycondensation [15], can also be carried out to obtain PPVs. Disadvantageously, only relatively low degrees of polymerization (up to 35) can be reached [15] and usually some of the metal organic catalyst remains in the polymer, which can cause shortcircuits and degradation of the photo-active material in the built devices [16]. Higher molecular weight material and metal-free PPVs are usually obtained using the so-called precursor routes [17]. They are characterized by the fact that, in the first step, precursor polymers (or prepolymers) are synthesized by adding understochiometric amounts of base [18] to (pre)monomers. These prepolymers are soluble in commonly used organic solvents, like THF or chloroform, and can be processed from these solvents easily. In the 
second step, the prepolymers are treated with an excess of base and/or heated, which converts the prepolymer into the fully conjugated PPV. Out of all precursor-routes, the so-called Gilch route [19] stands out, because it does not need complex premonomers and high temperatures to yield very high molecular weight polymers $\left(>10^{6} \mathrm{~g} / \mathrm{mol}\right)$ [20]. Due to the commercial availability of premonomers and their cheap and straight-forward synthesis, the Gilch polymerization is also used industrially to produce good quality PPVs [21].

Since the publication of the original paper by Gilch and Wheelwright in 1966 [19], a lot of research has been dealing with the reaction mechanism of the polymerization. The solvent and base used seem to play an important role and depending on the materials used, the mechanism can be radical and/or ionic. In the case of THF as the solvent and potassium tert-butoxide $\left(\mathrm{KO}^{t} \mathrm{Bu}\right)$ as base, it was shown that the polymerization proceeds via a radical chain growth mechanism, as it was verified by using spectroscopic methods (UV-Vis, NMR, EPR spectroscopy) and a radical trapping agent $[18,22,23]$. In Scheme 1 the reaction mechanism of alkoxy substituted $\alpha, \alpha^{\prime}$-dihalogenp-xylene 3 (premonomer) with $\mathrm{KO}^{t} \mathrm{Bu}$ is shown.

In the first step, a base induced 1,6-elimination of $\mathrm{HX}$ takes place at the premonomer 3 , which leads to the intermediate $\alpha$-halogen- $p$-quinodimethane 4 , which functions as the actual monomer. The radical reaction starting dimer 5 is generated by spontaneous dimerization of two p-quinodimethane $\mathbf{4}$ molecules. The dimerization only takes place in the shown constitution: the two aromatic units are bridged by an ethyl unit and the radicals are, due to radical stabilizing effects, located at the bromine bearing carbon atoms [24]. Subsequently the polymer grows toward the prepolymer 6 by adding $p$-quinodimethane 4 molecules at both chain ends. In the last step, another base induced HX-elimination takes place, which finally leads to the fully conjugated MEH-PPV 2.

\section{Synthesis of MEH-PPV via Gilch in the Research Laboratory}

To obtain high-quality polymers with good solubility, high molecular weight and low (constitutional) defect contents, the work group at the MPI for Polymer Research synthesizes PPVs typically at low temperatures ( -80 to $50^{\circ} \mathrm{C}$ ) [25]. The bromine functionalized version of premonomer $\mathbf{3}$ and $\mathrm{KO}^{t} \mathrm{Bu}$ are dissolved separately in THF. The use of non-stabilized THF is indicated, because the commonly deployed stabilizer BHT (butylated hydroxytoluene) is a radical scavenger, which might influence the radical polymerization. Additionally, the solvent is used in the dry and degassed state to avoid, on one hand, a side reaction of water with the base and, on the other hand, diradical oxygen to react with the radical chain ends. The premonomer solution is prepared in a three-necked-flask and cooled down to $-85^{\circ} \mathrm{C}$, while the flask is kept under argon or nitrogen, to avoid any ingress of humidity or air. The (prechilled) base solution is added slowly and then stirred for 8 hours, while the reaction mixture is allowed to warm up to room temperature. After an additional stirring time of 24 to $48 \mathrm{~h}$ at room temperature, the highly viscous polymer solution is precipitated in a fivefold excess methanol. The resulting fibrous solid is filtered off and redissolved in chloroform to remove the side products potassium bromide and tert-butanol as well as oligomers. After precipitating the polymer solution in methanol, filtering and drying in a drying oven at $40{ }^{\circ} \mathrm{C}$ and under reduced pressure, a compact red solid is obtained with $75-85 \%$ yield (with regard to the quantity of the used premonomer). Figure 3 shows a typical reaction setup and the reaction progress of the low temperature Gilch polymerization from the slightly yellow premonomer solution (left) towards the intensively red polymer solution (third from left).

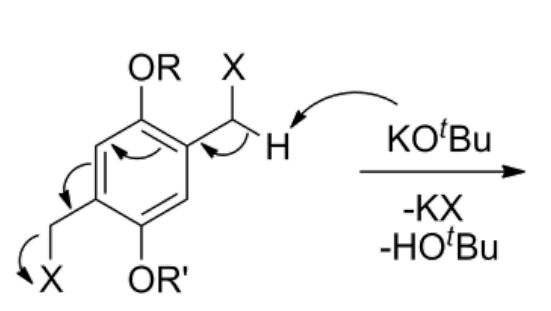

3

$$
\begin{aligned}
& \mathrm{X}=\mathrm{Cl}, \mathrm{Br} \\
& \mathrm{R}=\text { Methyl } \\
& \mathrm{R}^{\prime}=\text { Ethylhexyl }
\end{aligned}
$$

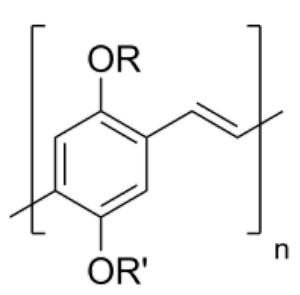

2<smiles>[Y]C=c1cc(O[R])c(=CC)cc1[R]</smiles>

4

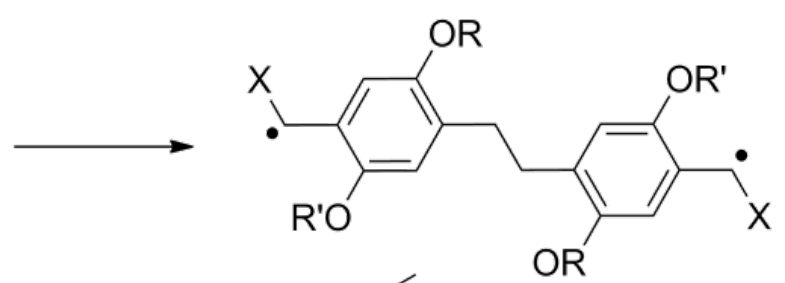

5

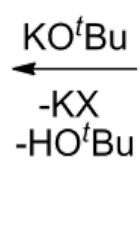

.

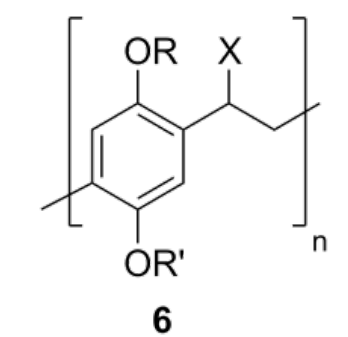

Prepolymer

\section{MEH-PPV}

Scheme 1. Radical chain growth mechanism of the Gilch precursor route of poly[2-methoxy-5-(2'-ethylhexyloxy)-1,4-phenylene vinylene] (MEH-PPV, 2) in THF 

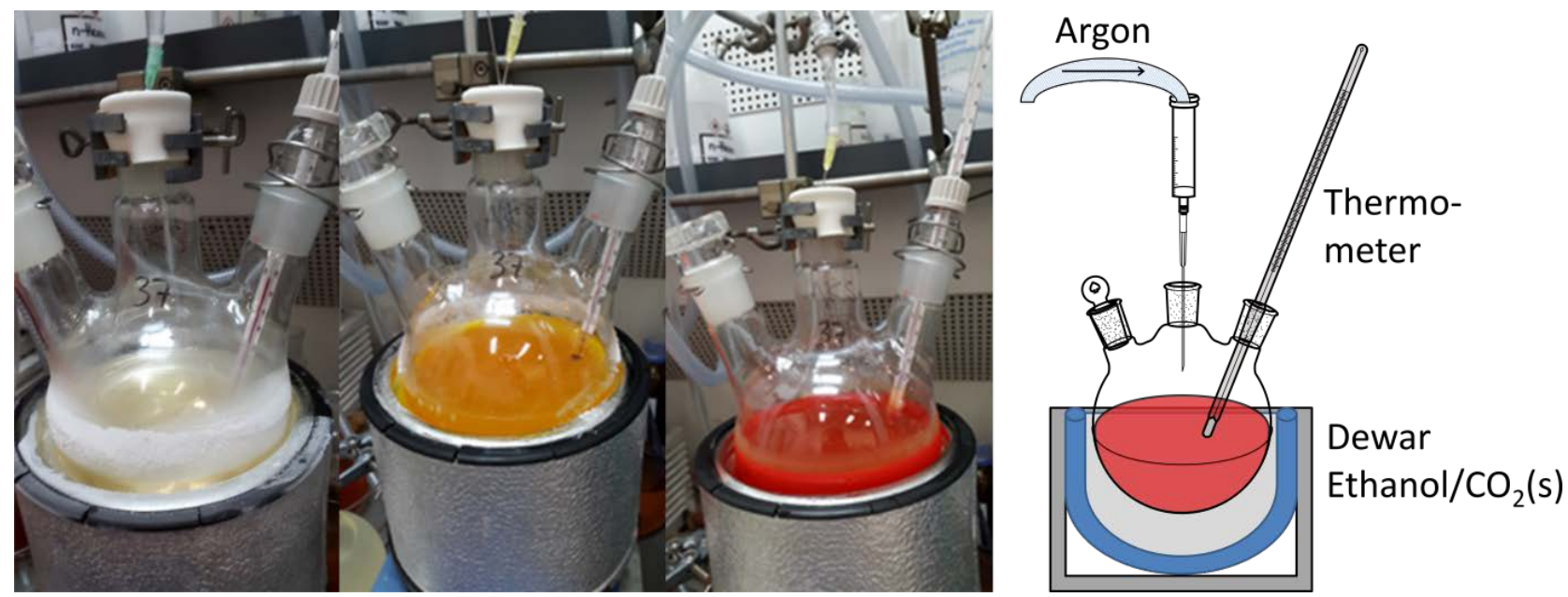

Figure 3. Exemplary reaction setup of the low temperature Gilch polymerization at different reaction times. The three-necked flask is equipped with an internal thermometer and an argon supply: Premonomer solution before addition of base (left), reaction mixture one hour after addition (first from left), reaction mixture $2.5 \mathrm{~h}$ after addition (second from left) and schematic representation of the reaction setup (right)

\section{Didactical Transformation}

We have made several didactical adjustments to optimize the synthesis of MEH-PPV to the needs of a school experiment. In Table 1, the most important modifications are summarized according to the factors "low-cost", "low-risk", "low-time" and "low-tech". The following section describes in detail the recent developments regarding the substitution of the solvent THF and the characterization of the products.

As mentioned above, the Gilch synthesis is typically carried out in THF, which is suspected to cause cancer. It is, therefore, advisable to replace this solvent with a less harmful one, especially when working with students. Ideally, the substitute has similar solubilizing quality as THF and is commercially available. MTBE (methyl tert-butyl ether) and m-THF (2-methyltetrahydrofuran) were investigated as the substitute for synthesis and OLED device fabrication. To ensure comparability with the THF experiments, all experimental parameters, such as concentrations of premonomer and base, stirring time and post-treatment of the obtained products were transferred to the MTBE/m-THF experiment.
The polymers synthesized in MTBE could not be dissolved sufficiently in standard solvents like THF or chloroform. Hence, the characterization of the products proved difficult. However, NMR spectra could be recorded (not shown here) and confirmed that the desired polymer (MEH-PPV) was obtained. The bad solubility of the material from the MTBE synthesis, even in good solvents, could not be explained up to now. After unsuccessful attempts to build OLEDs from this material, the idea of polymerization in MTBE was abandoned.

The second solvent, that was investigated, was 2-methyltetrahydrofuran (m-THF), which has also become an alternative for THF in organometallic chemistry in scientific research. It is not suspected to cause cancer and is not miscible with water. This marks another advantage of m-THF: conventional THF is fully miscible with water and is hygroscopic, which causes problems in the Gilch reactions under ambient conditions, because the base reacts with the absorbed water and is therefore not available for dehydrohalogenation of the premonomer. The solubilizing power of m-THF is expected to be very similar to the one of THF, which makes it a promising candidate as an alternative solvent for synthesis.

Table 1. Didactical adjustments for the Gilch-Synthesis of MEH-PPV

\begin{tabular}{|l|l|}
\hline Aspect & Didactical adjustments \\
\hline Low-cost & $\begin{array}{l}\text { The synthesis is being done in a small snap-cap vial instead of a three-necked-flask. This reduces the hazards, as well as the costs by } \\
\text { a factor of 10. As the complete headspace of the flask is filled with the solvent, there is no need of protection gas, as only minimal } \\
\text { amounts of oxygen and moisture can penetrate into the solution. Furthermore, we disclaim cooling, as the reaction occurs also at } \\
\text { room temperature and even at a higher speed. Although, the quality of the product is lower, we recommend these simplifications as } \\
\text { the polymer is still good enough to work in the low-cost OLED. }\end{array}$ \\
\hline Low-risk & $\begin{array}{l}\text { The Gilch-synthesis is usually done in the noxious and potentially carcinogenic solvent THF, which makes the experiment difficult } \\
\text { to handle in schools. We have succeeded in conducting the synthesis in 2-methyl-THF, which is neither toxic nor carcinogenic and } \\
\text { thus suitable for classroom-experiments. Furthermore, we substituted the toxic precipitation agent methanol by a mixture of ethanol } \\
\text { and water (7:3) for the precipitation of the polymer. Finally, we could reduce the amount of reactant and solvents by a factor of 10, } \\
\text { which makes the experiment safer in general. }\end{array}$ \\
\hline Low-time & $\begin{array}{l}\text { The duration of the different experimental steps (synthesis, precipitation, filtration, drying, etc.) was optimized sequentially. In this } \\
\text { way, we could reduce the total duration from 40 hours to only 60 min. Furthermore, we found a way to disclaim the drying process, } \\
\text { which additionally saves 12-24 hours of time. In this way, the synthesis of the polymer and the construction of the OLED can be } \\
\text { done within a standard class period (90 min). }\end{array}$ \\
\hline Low-tech & $\begin{array}{l}\text { The complexity of the experiment has been reduced by us, as we forgo multiple-necked-flasks, laboratory stands and protection gas. } \\
\text { Instead, we use snap-cap vials, in which we insert or extract the chemicals through syringes. This is easy to handle for students and } \\
\text { they can do the synthesis quickly and efficiently. Also, it is possible in this way to do multiple parallel syntheses using only one } \\
\text { fume-hood, which is important for schools with limited lab facilities. }\end{array}$ \\
\hline
\end{tabular}




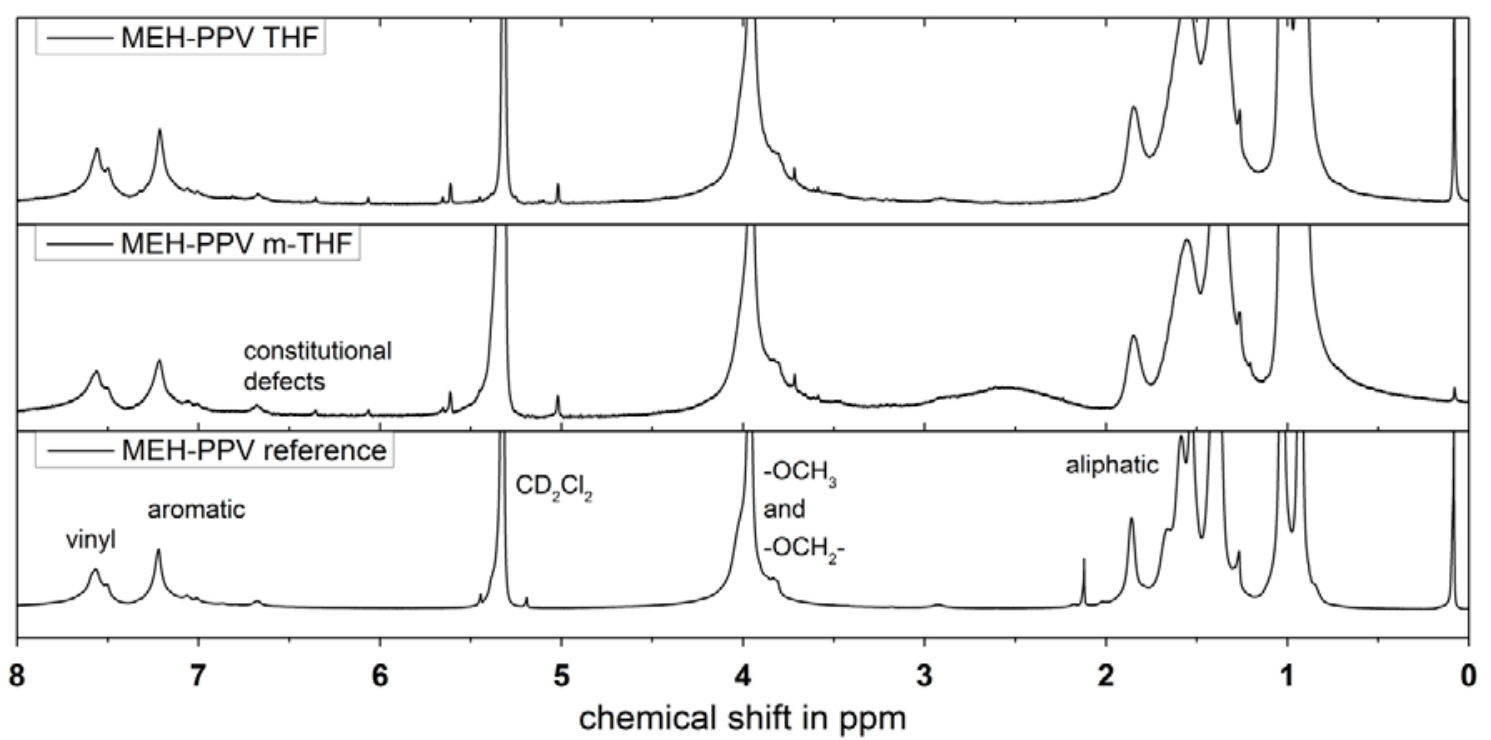

Figure 4. NMR spectra (300 MHz, $295 \mathrm{~K}$ in $\mathrm{CD}_{2} \mathrm{Cl}_{2}$ ) of three MEH-PPV samples synthesized in THF (top), m-THF (middle) and a reference sample from the scientific research laboratory (bottom). Important peaks for the functional groups and defects are assigned

The polymerization behavior in m-THF did not differ at all from the one in THF and a red solid was obtained after precipitation. The solubility of the product in commonly used solvents, like toluene, THF and dichloromethane, was very good. NMR-spectra of a representative "THF" and " $m$-THF" polymer and a reference sample are shown in Figure 4. The spectra agree well with the reference spectrum, despite a little higher defect content in the range between 5.5 and $7.0 \mathrm{ppm}$. These constitutional defects most likely originate from the higher reaction temperature. Since the reference sample was synthesized at low temperature, the number of constitutional defects is lower. In the m-THF polymer spectrum, a broad peak at $2.5 \mathrm{ppm}$ can be found. It could not be clarified where it comes from, one possible explanation might be a water peak (from the NMR solvent $\mathrm{CD}_{2} \mathrm{Cl}_{2}$ ), which is broadened due to residual base causing a $\mathrm{pH}$ change.

After the analysis via NMR, additional GPC measurements were carried out to investigate the molecular weight of the obtained polymers. In Figure 5, selected molecular weight distributions are displayed.

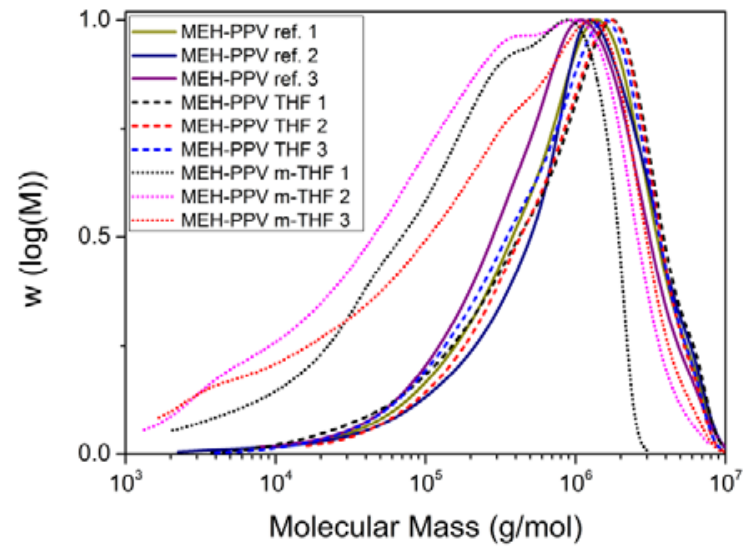

Figure 5. Molecular weight distributions of selected polymers, measured against a polystyrene calibration in THF. Reference samples were synthesized in the research laboratory in Mainz (straight lines), THF (short dashed) and m-THF (short dot) samples were obtained from the "quick and easy" synthesis procedure
According to Figure 5, all Gilch polymerizations lead to relatively broad distributions and high molecular weight polymers. The reference and "THF" sample curves are monomodal, almost on top of each other and show that the "quickly and easily" synthesized polymers are (reproducibly) as big as the ones from the research lab. The m-THF polymer curves have a slightly different shape. They are bimodal, which means that the curve has a prominent shoulder at lower molecular weights. This can also be seen in the values for $M_{\mathrm{n}}$ in Table 2: the number average molecular weights $M_{\mathrm{n}}$ of the m-THF samples are consistently much smaller than the others. This also leads to a higher dispersity $\oslash$, which is defined as the ratio of $M_{\mathrm{w}} / M_{\mathrm{n}}$ and describes the uniformity of polymers. Though, it should be noted that significant parts of the measured samples are of high molecular weight, having the absolute maximum of the distributions at $10^{6} \mathrm{~g} / \mathrm{mol}$ for all three $\mathrm{m}$ THF samples, outlining reproducibility of the experiment. The origin of the low molecular weight tail is not known yet and is still under investigation.

Table 2. Weight $\left(M_{\mathrm{w}}\right)$ and number average molecular weights $\left(M_{\mathrm{n}}\right)$, as well as dispersities $\boldsymbol{D}$ of polymer samples obtained from different syntheses, measured in THF at $30^{\circ} \mathrm{C}$ against a polystyrene calibration. The naming corresponds to the samples presented in Figure 5

\begin{tabular}{|c|c|c|c|}
\hline & $M_{\mathrm{w}}$ in g/mol & $M_{\mathrm{n}}$ in g/mol & $D$ \\
\hline MEH-PPV ref. 1 & 1470400 & 314170 & 4.68 \\
\hline MEH-PPV ref.2 & 1595900 & 243540 & 6.55 \\
\hline MEH-PPV ref.3 & 1302700 & 291380 & 4.47 \\
\hline MEH-PPV THF 1 & 1558620 & 252711 & 6.17 \\
\hline MEH-PPV THF 2 & 1536480 & 413252 & 3.72 \\
\hline MEH-PPV THF 3 & 1439490 & 288683 & 4.99 \\
\hline MEH-PPV m-THF 1 & 508366 & 56133 & 9.06 \\
\hline MEH-PPV m-THF 2 & 666938 & 36433 & 18.31 \\
\hline MEH-PPV m-THF 3 & 845646 & 38942 & 21.72 \\
\hline
\end{tabular}




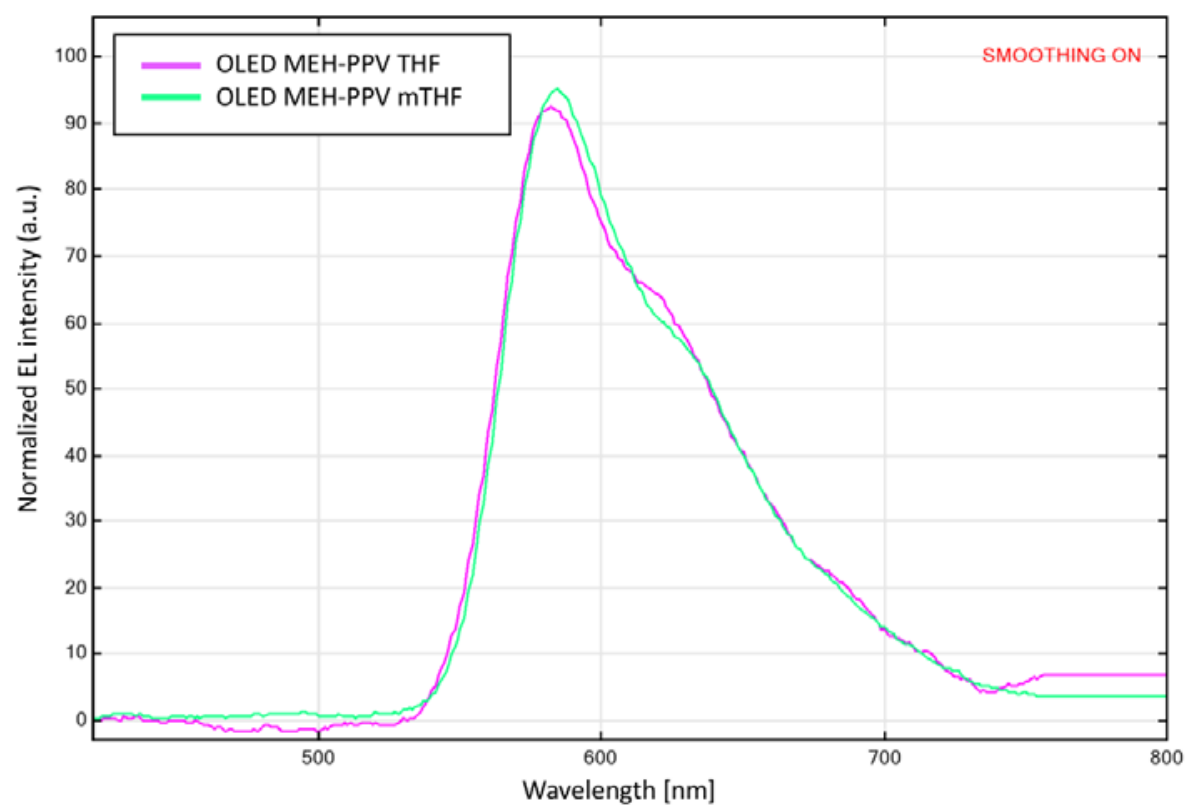

Figure 6. Electroluminescence spectra of the OLEDs with MEH-PPV synthesized in THF (pink) and m-THF (green)

To investigate the electroluminescence (EL) quality of the polymers, the materials have been incorporated into low-cost OLED devices, from which EL-spectra were recorded. The spectra in Figure 6 clearly show that the EL-spectra are quite independent from the solvent, in which the polymer was synthesized. The emission maxima of both the polymers are very close to each other (581 nm for the THF and $583 \mathrm{~nm}$ for the m-THF polymer).

Despite the differences in overall molecular weights, dispersities and EL characteristics, the "m-THF polymers" are soluble and big enough to obtain good quality films showing bright luminescence when applied in the OLED. Therefore, $\mathrm{m}$-THF is proposed as an alternative solvent for the MEH-PPV synthesis for students. The following experimental part describes the "quick and easy" synthesis of MEH-PPV in detail.

\section{Experimental Part}

\subsection{Experiment 1: Synthesis of MEH-PPV in a Quick \& Easy Experiment}

Safety instructions: The syntheses have to be conducted under a fume hood, wearing gloves, a lab coat and safety glasses. The syringes must be handled with care!

Materials: 2 snap-cap vials $(5 \mathrm{~mL}), 2$ small and 1 medium magnetic stirring rods, 2 syringes with needles (1 $\mathrm{mL} \& 10 \mathrm{~mL}$ ), $150 \mathrm{~mL}$ beaker, $250 \mathrm{~mL}$ Erlenmeyer flask, magnetic stirrer, drying oven, funnel, filter paper, small watch glass, aluminium foil, spatula

Chemicals:

- Premonomer: 1,4-Bis(bromomethyl)-2-((2'ethylhexyl)-oxy)-5-methoxybenzene, (C, GHS05), CAS: 209625-37-6

- Base: Potassium tert-butoxide, (F, GHS02), (C, GHS05), CAS: 865-47-4

- m-THF: 2-Methyltetrahydrofuran with 250 ppm BHT for stabilization, (F, GHS02), (Xi, GHS07), (Xn, GHS08), CAS: 109-99-9
- Ethanol techn. grade, (F, GHS02), (Xi, GHS07)

1. Fill $0.04 \mathrm{~g}$ of the premonomer into the first vial and $0.08 \mathrm{~g}$ of the base into the second vial. Add a small stirring rod to each vial and close them with the caps (Figure 7 - left). Now, stitch 2 holes into each cap with the help of a needle and add $4 \mathrm{~mL}$ of m-THF to each vial using the $1 \mathrm{~mL}$-syringe (use only the holes and don't remove the caps). Let the mixtures stir for about $5 \mathrm{~min}$ (Figure 7- center) on the magnetic stirrer.

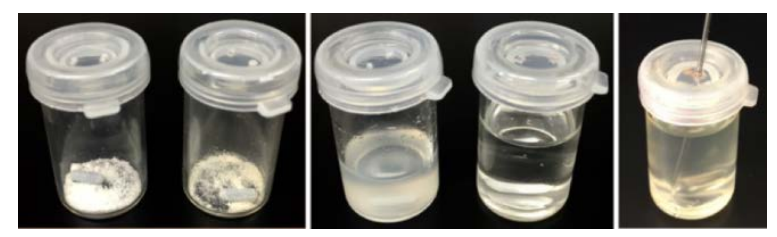

Figure 7. Solid reactants (left); dissolved reactants (center); solutions are merged (right). Reprinted with permission [4]

2. Add the premonomer solution to the base solution using the 10mL-syringe (Figure 7, right). Note: Any remaining headspace must be filled with m-THF to minimize the influence of oxygen and humidity. The solution has to be stirred for around $15 \mathrm{~min}$. When the colour changes from yellow to orange (Figure 8), cover the vial with a piece of aluminium foil to protect it from ambient-light.

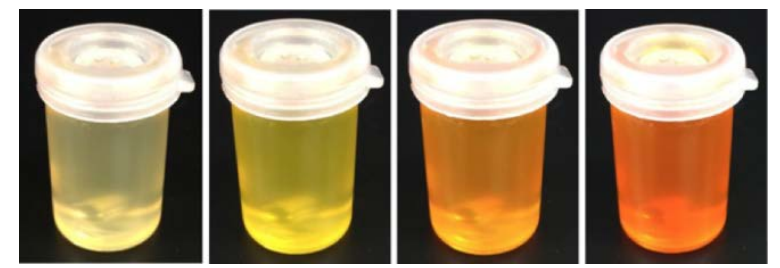

Figure 8. Color change during the synthesis. Reprinted with permission [4]

3. Place a beaker with $20 \mathrm{~mL}$ ethanol/water (7:3) on a magnetic stirrer. Use the $10 \mathrm{~mL}$-syringe to transfer the content of the vial slowly into the beaker to precipitate the polymer (Figure 9, left). Now stir the mixture for another 5 min and then filter it (Figure 9, 1. f. left). 
4. Scrape off the moist polymer from the filter paper and transfer it onto a watch glass (Figure 9, upper-right). Cover it with perforated aluminium foil and dry the polymer overnight in a drying oven at $40^{\circ} \mathrm{C}$.
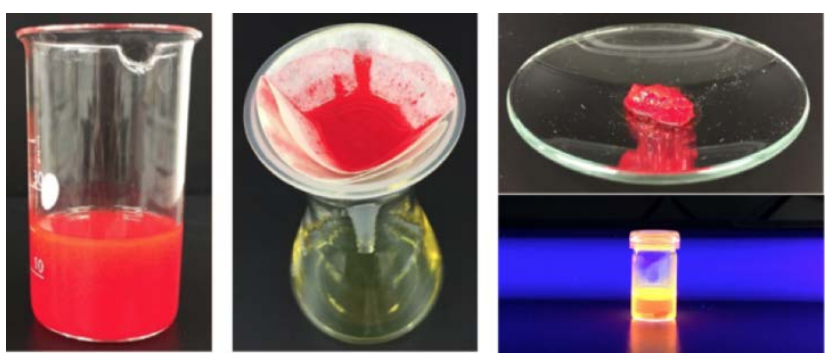

Figure 9. Precipitation of the polymer (left); filtration of the polymer (1. f. left); moist polymer on a watch glass (upper-right); fluorescence of the MEH-PPV solution under UV light (lower-right). Reprinted with permission [4]

5. Preparing the MEH-PPV-solution for the OLED (method 1):

The dried polymer has to be dissolved in m-THF before spincoating. The necessary mass concentration is $\beta=3$ $\mathrm{mg} / \mathrm{mL}$. Stir the solution overnight at $50{ }^{\circ} \mathrm{C}$ to ensure that the polymer has been dissolved completely. The MEHPPV-solution shows a strong orange colored fluorescence under UV light (Figure 9, lower-right) and should be stored in a dark and cold place (i.e. fridge).

6. Preparing the MEH-PPV-solution for the OLED (method 2):

To spare time, you can alternatively skip the drying process and directly dissolve the moist polymer in m-THF. The weight percentage of the polymer in the wet product is $\sim 3 \%$. To obtain a solution with $\beta=3 \mathrm{mg} / \mathrm{mL}$, add $1 \mathrm{~mL}$ m-THF per $1 \mathrm{mg}$ polymer. Note that due to impurity and remaining precipitating agent, the functionality of the resulting OLEDs may be worse compared to those made via method 1.

\subsection{Experiment 2: Construction of the Low-cost OLED with the Self-synthesized MEH-PPV}

Safety-instructions: Spincoating must be done under the fume-hood wearing safety-glasses, a lab coat and gloves.

Materials: 1 FTO (fluorine tin oxide) glass slide (3.5 x $3.5 \mathrm{~cm})$, microscope slide, 2 pipette, hairdryer, PC ventilator (low-cost Spincoater), PET-bottle neck for splash protection, $1 \mathrm{~mL}$ syringe with needle, Galinstan (gallium-indium-tin-alloy), 1 Galinstan mask, 2 foldback-clips, multimeter, DC power source $(0-12 \mathrm{~V}), 2$ connection cables, 2 crocodile cable, tissue paper, sellotape Chemicals:

- MEH-PPV-solution $(\beta=3 \mathrm{mg} / \mathrm{mL})$; Poly[2-methoxy5-(2-ethylhexyloxy)-1,4-phenylene vinylene], (F, GHS02), (Xi, GHS07), (Xn, GHS08), self-made from experiment 1

- PEDOT: PSS; Poly(3,4-ethylendioxythiophene): Poly(styrenesulfonate) 1.3-1.7 w\% aqueous dispersion, (Xi, GHS07), CAS: 155090-83-8

- Acetone tech. grade, (F, GHS02), (Xi, GHS07), CAS: 67-64-1

Please refer to our video-tutorial [26] (QR code), where you can observe each building step in detail. Note: The polymer used in the videos is Superyellow instead of $M E H-P P V$. The experimental steps are the same for both polymers.

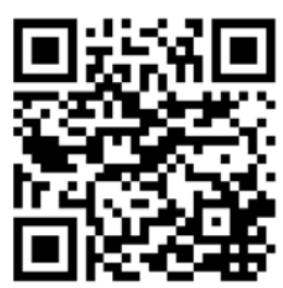

QR-Code for videos

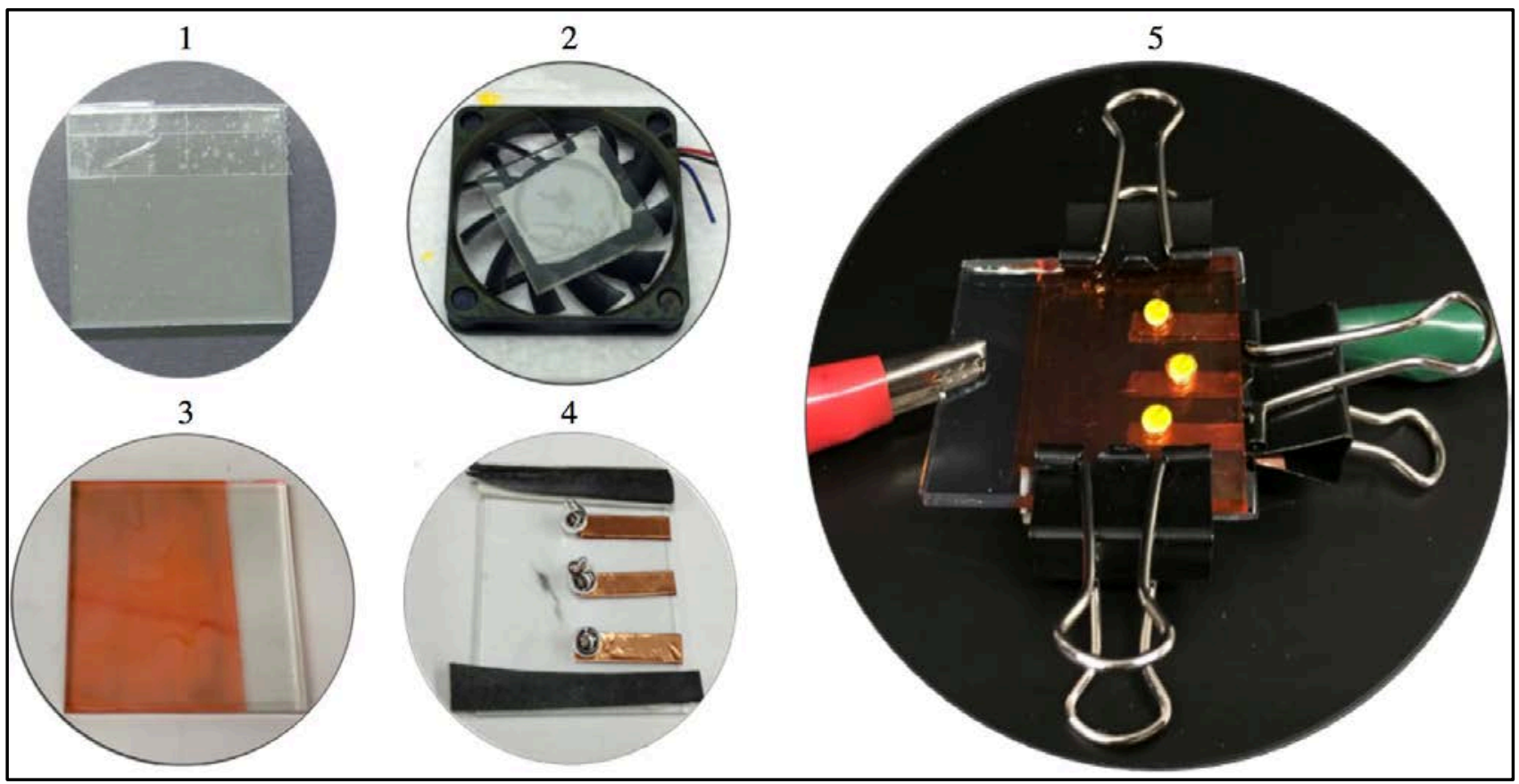

Figure 10. Picture series of the low-cost OLED-construction with MEH-PPV.1) Prepared FTO-glass, 2) PC-fan as low-cost spincoater, 3) FTO glass coated with MEH-PPV,4) Cathode mask with Galinstan drops, 5) Electroluminescence of the MEH-PPV-OLED at 9 V.Reprinted with permission [4] 
1. Clean the FTO slide with acetone and a tissue paper. Determine the conductive side of the glass using a multimeter and attach a stripe of sellotape to the conductive side (Figure 10000-1). Put a few drops of the conductive polymer PEDOT:PSS on the glass and wipe the liquid off from the glass surface with a microscope slide. Dry the layer with a hair-dryer. (Note: PEDOT:PSS increases the brightness of the OLED.)

2. Attach the FTO glass onto the PC fan with a piece of double sided tape (Figure 10000-2). Connect the fan to the DC power supply and apply 4.5 V. Put the splash protection over the Spincoater and inject $0.1 \mathrm{~mL}$ of the MEH-PPV-solution onto the rotating glass. Wait for 15 sec. and then remove the glass. You should get a thin orange-red colored layer of the polymer on your glass surface (Figure 10000-3). If you spot holes in the layer, you need to spincoat a second layer on the top of the first. Remove the stripe of sello tape from step 1.

3. The liquid alloy Galinstan is used as the cathode material. To hold the alloy in place, a special low-cost mask is used, made from a microscope slide equipped with rubber-spacers and copper-strips. Place three drops of Galinstan (in the size of a pinhead) onto each copper-strip (Figure 10000-4) and then put the coated FTO-glass on top of the mask (Figure 10000-5) resulting in a smooth contact between MEH-PPV and Galinstan. Fix the device with the foldback-clips.

4. Connect the OLED to the DC-power supply (FTO = +; Galinstan = -). Increase the voltage stepwise until the OLED starts to glow (approx. at $5 \mathrm{~V}$ ). By increasing the voltage further, the intensity of the luminescence can be increased as well. Avoid higher voltages (>15 V), as the OLED will start to show short-circuit sparks and the polymer layer will be destroyed.

\section{Summary \& Conclusion}

If we want to motivate students towards STEM (science, technology, engineering and mathematics), it is crucial to show them the relevance of STEM for their everyday life, the environment or mankind. The implementation of modern and future-relevant topics from current science research into the science curriculum of educational institutions is a promising way to achieve this goal. The development of "school tailored" experiments is one of the most important factors for a successful implementation.

The aim of this work was to transform the Gilch synthesis of MEH-PPV to the needs of a didactical experiment. Following this aim, we have modified the experimental parameters for the Gilch synthesis in terms of lower costs, lower risks, lower time and less technical requirements. The achieved quick \& easy Gilch synthesis of MEH-PPV does not need protection gas and is conducted in a snap-cap-vial using the less harmful solvent m-THF (instead of the standard and potentially carcinogenic solvent THF). The duration of the whole experiment was reduced from 40 hours to $60 \mathrm{~min}$. NMR- and GPCmeasurements proved, that the quick \& easy synthesis leads to polymers with reproducible good quality and high molecular masses. Furthermore, we could incorporate the self-made MEH-PPV into our low-cost OLEDs, resulting in bright orange-red electroluminescent devices.
The development of new didactical experiments from current research needs a close cooperation with colleagues from the subject sciences. Also this work would not have been possible without the intensive guidance and support from the MPI, where - for instance - all NMR- and GPCmeasurements have been recorded and interpreted.

The next step of our research is to test the developments along with teachers and students in a Design-BasedResearch [27] approach. Furthermore, we are working on the quick \& easy Gilch synthesis of MDMO-PPV (Poly[2methoxy-5-(3',7'-dimethyloctyloxy)-1,4-phenylene vinylene]), which is an effective (donor) material for (bulk heterojunction) organic solar cells.

\section{References}

[1] Banerji, A.; Tausch, M. W.; Scherf, U., "Fantastic Plastic". CHEMKON, 19 (1), 7-12, 2012.

[2] Banerji, A., "Organische Elektronik als Lehrstoff". Nachrichten aus der Chemie, 65 (7-8), 807-809, 2017.

[3] Banerji, A.; Dörschelln, J.; Schwarz, D., "Organische Leuchtdioden im Chemieunterricht". Chemie in unserer Zeit, in press.

[4] Banerji, A.; Schönbein, A. K.; Wolff, J., "OLED Reloaded: Die Synthese des Halbleiterpolymers MEH - PPV als Schulversuch". CHEMKON, 24 (4), 251-256, 2017.

[5] Heeger, A. J., "Semiconducting and metallic polymers: the fourth generation of polymeric materials (Nobel lecture)". Angewandte Chemie International Edition, 40 (14), 2591-2611, 2001.

[6] Shirakawa, H.; Louis, E. J.; MacDiarmid, A. G.; Chiang, C. K.; Heeger, A. J., "Synthesis of electrically conducting organic polymers: halogen derivatives of polyacetylene, (CH)". Journal of the Chemical Society, Chemical Communications, (16), 578-580, 1977.

[7] Roncali, J., "Molecular Engineering of the Band Gap of $\pi$-Conjugated Systems: Facing Technological Applications". Macromolecular Rapid Communications, 28 (17), 1761-1775, 2007.

[8] Yang, X.; Neher, D., "Polymer electrophosphorescence devices". Organic Light Emitting Devices: Synthesis, Properties and Applications, 333-367, 2006.

[9] Tang, C. W.; VanSlyke, S., "Organic electroluminescent diodes". Applied Physics Letters, 51 (12), 913-915, 1987.

[10] Adachi, C.; Tsutsui, T.; Saito, S., "Blue light - emitting organic electroluminescent devices". Applied Physics Letters, 56 (9), 799801, 1990.

[11] Søndergaard, R. R.; Hösel, M.; Krebs, F. C., "Roll - to - Roll fabrication of large area functional organic materials". Journal of Polymer Science Part B: Polymer Physics, 51 (1), 16-34, 2013.

[12] Burroughes, J. H.; Bradley, D. D. C.; Brown, A. R.; Marks, R. N.; Mackay, K.; Friend, R. H.; Burns, P. L.; Holmes, A. B., "Lightemitting diodes based on conjugated polymers". Nature, 347 (6293), 539-541, 1990.

[13] Fink, J. K., High Performance Polymers. William Andrew: 2014.

[14] McDonald, R. N.; Campbell, T. W., "The Wittig Reaction as a Polymerization Method1a". Journal of the American Chemical Society, 82 (17), 4669-4671, 1960.

[15] Koch, A.; Harrison, N.; Haylett, N.; Daik, R.; Feast, W.; Friend, R., "Enhanced photostability of poly (1, 3-phenylene diphenylvinylene)-derivatives by diphenyl-substitution". Synthetic Metals, 100 (1), 113-122, 1999.

[16] Krebs, F. C.; Nyberg, R. B.; Jørgensen, M., "Influence of residual catalyst on the properties of conjugated polyphenylenevinylene materials: palladium nanoparticles and poor electrical performance". Chemistry of Materials, 16 (7), 1313-1318, 2004.

[17] Junkers, T.; Vandenbergh, J.; Adriaensens, P.; Lutsen, L.; Vanderzande, D., "Synthesis of poly (p-phenylene vinylene) materials via the precursor routes". Polymer Chemistry, 3 (2), 275-285, 2012.

[18] Schwalm, T. Studien zum Mechanismus der GILCHPolymerisation und zum Ermüdungsverhalten von Poly (pphenylen-vinylen)en (PPVs) in organischen lichtemittierenden Dioden (OLEDs). Dissertation, TU Darmstadt, 2009. 
[19] Gilch, H.; Wheelwright, W., "Polymerization of $\alpha$ - halogenated p - xylenes with base". Journal of Polymer Science Part A: Polymer Chemistry, 4 (6), 1337-1349, 1966.

[20] Becker, H.; Spreitzer, H.; Ibrom, K.; Kreuder, W., "New Insights into the Microstructure of GILCH-Polymerized PPVs". Macromolecules, 32 (15), 4925-4932, 1999.

[21] Roex, H.; Adriaensens, P.; Vanderzande, D.; Gelan, J., "Identification and Quantification of Polymerization Defects in 13C-Labeled Sulfinyl and Gilch OC1C10-PPV by NMR Spectroscopy". Macromolecules, 36 (15), 5613-5622, 2003.

[22] Schwalm, T.; Wiesecke, J.; Immel, S.; Rehahn, M., "Toward Controlled Gilch Synthesis of Poly(p-phenylene vinylenes): Anionic vs Radical Chain Propagation, a Mechanistic Reinvestigation". Macromolecules, 40 (25), 8842-8854, 2007.

[23] Schwalm, T.; Wiesecke, J.; Immel, S.; Rehahn, M., "The Gilch Synthesis of Poly(p-phenylene vinylenes): Mechanistic Knowledge in the Service of Advanced Materials". Macromolecular Rapid Communications, 30 (15), 1295-1322, 2009.

[24] Wiesecke, J.; Rehahn, M., "[2.2]Paracyclophanes with Defined Substitution Pattern-Key Compounds for the Mechanistic Understanding of the Gilch Reaction to Poly(p-phenylene vinylene)s". Angewandte Chemie International Edition, 42 (5), 567-570, 2003.

[25] Schönbein, A.-K.; Wagner, M.; Blom, P. W. M.; Michels, J. J., "Quantifying the Kinetics of the Gilch Polymerization toward Alkoxy-Substituted Poly(p-phenylene vinylene)". Macromolecules, 50 (13), 4952-4961, 2017.

[26] Banerji, A., http://www.chemiedidaktik.uni-koeln.de/oled.html, last accessed: 15.12.2017.

[27] Design-Based-Research Collective. Educational Researcher, 32(1), 2003 